\title{
MicroRNA-mediated iPS cells
}

\section{By Kai-Jye Lou, Staff Writer}

The standard protocol for reverting somatic cells into induced pluripotent stem cells with viral vectors that introduce a quartet of transcription factors has low reprogramming efficiency. ${ }^{1}$ Moreover, some human cell types are refractory to reprogramming with transcription factors. Now, researchers at the University of Pennsylvania report that microRNAs could address both problems, taking exogenous transcription factors out of the picture while increasing reprogramming efficiency. ${ }^{2}$

The UPenn protocol still uses viral vectors, which could pose safety issues in the clinic. Thus, the group now needs to replicate the findings using nonviral delivery of the miRNAs.

In 2006, Japanese researchers published the first protocol for reprogramming somatic cells into induced pluripotent stem (iPS) cells using retroviral vectormediated expression of the transcription factors Oct4, Sox2, Klf4 and c-Myc. Reprogramming efficiencies using the method range from $0.2 \%-0.8 \% .^{3}$

The exogenous delivery of transcription factor proteins plus cocktails of small molecules could supplant viral vectors, but such protocols have even lower reprogramming efficiency. Also, the need for a steady supply of a transcription factor protein would not be cost effective.

"I think high reprogramming efficiencies would be necessary for developing a reliable high throughput platform for generating iPS cells," said Edward Morrisey, a professor of medicine at UPenn and scientific director of the Penn Institute for Regenerative Medicine.

According to Morrisey, many types of human cells are refractory to reprogramming by using the transcription factors. Thus, his group at UPenn looked to miRNAs to address both efficiency and cell type issues.

Recent studies showed that miRNAs can increase the efficiency of reprogramming with transcription factors. ${ }^{4-7}$

The UPenn researchers went a step further and showed that lentiviral-mediated expression of the miRNA-302/367 cluster of miRNAs reprogrammed mouse embryonic fibroblasts into iPS cells with higher efficiency than the standard four transcription factor reprogramming protocol. At 8 and 10 days following viral transduction, cell counting showed that the miRNA reprogramming protocol generated about two orders of magnitude more iPS cells than the standard protocol. Similar results were seen in human fibroblasts.

Results were published in Cell Stem Cell.

"Our work shows that you could use miRNAs to efficiently reprogram both mouse and human somatic cells into induced pluripotent stem cells without delivering any of the four transcription factors that are usually needed for efficient reprogramming," said Morrisey, who is corresponding author on the paper. He added that the miRNAs also might be useful in reprogramming human cells that are refractory to the quartet of transcription factors.

"This certainly is a very interesting result, as previous studies have only shown that miRNAs can facilitate and replace some of the transcription factors used for reprogramming these cells," said Sheng Ding, a senior investigator at the Gladstone Institute of Cardiovascular Disease and a professor in the Department of Pharmaceutical Chemistry at the University of California, San Francisco. Ding is a scientific cofounder of Fate Therapeutics Inc., which is focused on developing stem cell therapeutics.

"The increase in somatic cell reprogramming efficiency with this new technique is a significant advancement," said Kuldip Sidhu, an associate professor and director of the stem cell lab at The University of New South Wales. "This is very important, as reprogramming efficiency with small molecules, proteins and peptides continues to be very low, and the associated procedures can be cumbersome as the continuous presence of such factors is required for extended periods. Such methods are currently not very cost effective."

\section{Delivering the goods}

Morrisey said one possible reason for the success of his team's protocol could be that in addition to reprogramming, miRNAs may be able to promote cell proliferation, which would help speed up the generation of iPS cells. His group now is working to better understand the mechanisms behind miRNA-mediated reprogramming.

Other researchers want to see the reprogramming protocol repeated using nonviral vectors. Viral vectors can cause genomic insertions, which could raise safety concerns in clinical trials of a cell therapy derived from such iPS cells.

"Ultimately, they will need to demonstrate that their approach works with a nonintegrating vector before applications in a practical |setting could be realized," said Ding. "They will need to show that they could achieve these results by actually delivering the miRNAs themselves into the cell."

"Even though the researchers used viral vectors in their current study, they should be able to use nonviral methods like miRNA mimics to deliver the same miRNAs to the cell," said Robert Blelloch, an associate professor in the Department of Urology and member of the Eli and Edythe Broad Center of Regeneration Medicine and Stem Cell Research at UCSF. "We and others have already shown that miRNA 


\section{ANALYSIS/PROSPECTS}

mimics can be efficiently transfected into cells to promote reprogramming," with transcription factors.

Morrisey added that episomal plasmids, which can replicate within cells for a while and do not integrate into the genome, are another possible vector for miRNA delivery.

"We are interested in trying to adapt our method into a robust and simple protocol using nonviral vectors," he told SciBX.

UPenn has filed a patent application covering the technology, which is available for licensing.

Lou, K.-J. SciBX 4(18); doi:10.1038/scibx.2011.504

Published online May 5, 2011

\section{REFERENCES}

1. Takahashi, K. \& Yamanaka, S. Cell 126, 663-676 (2006)
2. Anokye-Danso, F. et al. Cell Stem Cell; published online April 8, 2011; doi:10.1016/j.stem.2011.03.001

Contact: Edward E. Morrisey, University of Pennsylvania, Philadelphia, Pa. e-mail: emorrise@mail.med.upenn.edu

3. Huangfu, D. et al. Nat. Biotechnol. 26, 795-797 (2008)

4. Subramanyam, D. et al. Nat. Biotechnol.; published online April 13, 2011; doi:10.1038/nbt.1862

5. Liao, B. et al. J. Biol. Chem.; published online March 22, 2011; doi:10.1074/jbc.C111.235960

6. Li, Z. et al. EMBO J. 30, 823-834 (2011)

7. Judson, R.L. et al. Nat. Biotechnol. 27, 459-461 (2009)

COMPANIES AND INSTITUTIONS MENTIONED

Fate Therapeutics Inc., San Diego, Calif.

Gladstone Institute of Cardiovascular Disease, San Francisco, Calif. University of California, San Francisco, Calif.

The University of New South Wales, Sydney, New South Wales, Australia University of Pennsylvania, Philadelphia, $\mathrm{Pa}$. 